\title{
Gestalt in Coaching und Beratung
}

\section{T. Bachmann • H. Möller}

Online publiziert: 29. Juli 2020

(C) Der/die Autor(en) 2020

Vor fast genau 70 Jahren, 1951, erschien das erste Buch zur Gestalttherapie - ,Excitement und Growth in Human Personality“ stand im Untertitel. Für dieses gemeinsame Projekt schöpften Fritz und Lore Perls, Paul Goodman und Ralph Hefferline aus Quellen der Gestaltpsychologie, des Existenzialismus, der Psychoanalyse, aus fernöstlichen Lehren und körperorientierten Ansätzen, wie sie z. B. Wilhelm Reich vertrat. Später wurden Bubers Dialogphilosophie und Lewins Feldtheorie integriert. Kaum ein sonstiger therapeutischer Ansatz strebt so reichhaltig nach Ganzheitlichkeit, hatte und hat er doch den Anspruch, den Menschen in seinem inneren und äußeren Verwobensein mit anderen und mit der Umwelt zu begreifen und ihn zu ermutigen, für seine Bedürfnisse, Wünsche und Träume einzutreten. Dieses Anliegen ist bis heute Leitbild der Gestalttherapie, wenn inzwischen auch differenzierter und nicht mehr so radikal wie in den Anfangsjahren, in denen jede Überlegung, jedes Denken als Kontaktstörung bezeichnet wurden, weil es die spontanen Impulse hemmte. Vergleicht man die Gestalttherapie mit anderen Ansätzen, fällt auf, dass sie kaum über so etwas wie Methoden oder Techniken verfügt. Ihre Kraft und Faszination steckt in der Haltung des Therapeuten und den grundlegenden Arbeitsprinzipien. In einer gestalttherapeutischen Sitzung begegnet der Therapeut dem Klienten mit ganzer Präsenz, umfassend und bestätigend und als Dialogpartner im Buberschen Sinne. Das „Hier und jetzt“ (nicht das „Dann und dort“) steht im Mittelpunkt, die Sitzung wird zum Erfahrungsraum und Experimentierfeld für den Klienten. Ziel ist nicht die Veränderung, sondern die Förderung von Gewahrsein und Bewusstheit, mit

PD Dr. T. Bachmann

artop GmbH, Christburger Str. 4, 10405 Berlin, Deutschland

E-Mail: bachmann@artop.de

Prof. Dr. H. Möller $(\bowtie)$

Institut für Psychologie, Universität Kassel, Holländische Straße 36-38, 34127 Kassel, Deutschland

E-Mail: heidi.moeller@uni-kassel.de 
der Gewissheit, dass diese ganz von selbst für Veränderung und Wachstum sorgen wird.

Der Erfolg der Gestalttherapie besteht nicht nur darin, dass sie sich seitdem als wichtige Therapierichtig etabliert hat, sondern in ihren theoretischen und praktischen Einflüssen z.B. auf die Systemtheorie, die Motivations-, Persönlichkeits-, Lern- und Entwicklungs-, Organisations- und Arbeitspsychologie sowie die praktischen Anwendungen in Coaching, Organisationsberatung und Change-Management. Die „offene Gestalt“, Achtsamkeit und „Awareness“, Orientierung an eigenen Bedürfnissen und Wünschen, Selbstsorge, Selbstausdruck, Intuition, Kontaktgestaltung, Begegnung, aber auch Veränderungsansätze wie Lewins „Unfreeze-ChangeRefreeze“ oder das in letzter Zeit vielfach beachtete Konzept der „Psychological Safety" haben - neben vielen anderen Impulsen - ihren Ursprung in Gestaltansätzen. In den Artikeln zu diesem Themenschwerpunkt ist eine kleine Auswahl von Perspektiven und Anwendungen des Gestaltansatzes zusammengestellt.

Im Artikel von Wolfang Looss wird zunächst das Spannungsfeld zwischen dem personenorientierten Gestaltansatz und dessen Anwendung auf Organisationen als soziale Systeme diskutiert. Die Denkfigur des organisationalen Selbst wird erforscht, und schließlich werden praktische Beobachtungsmöglichkeiten für Beratungspersonen vorgestellt und erläutert.

Thomas Bachmann untersucht anhand des Konzepts der Kontaktgrenze aktuelle gesellschaftliche Entwicklungen und deren Auswirkungen auf das Coaching. Der Artikel lädt zum Nachdenken und Mit-Philosophieren über die Veränderungen der Kontaktgrenzen ein: der eigenen, mit deren Hilfe wir unser Leben gestalten, ihrer inneren Repräsentation, die uns Halt und Orientierung gibt, und der Kontaktgrenzen zwischen Systemen und Subsystemen der Gesellschaft, die deren Zusammenspiel charakterisieren.

Monika Stützle-Hebel wendet in ihrem Artikel über Agiles Arbeiten das Lebensraumkonzept aus der Feldtheorie Kurt Lewins praktisch auf dieses dynamische Umfeld an. Mehrfachzugehörigkeiten, Gleichzeitigkeit, Geschwindigkeit, Konflikte und andere dynamisierende Parameter werden untersucht und deren Auswirkungen auf die Lebensraumrepräsentation von Teams und ihren Mitgliedern beschrieben. Daraus leitet die Autorin konkrete und praktische Ansatzpunkte für das Coaching agiler Teams ab.

Der Schwerpunktteil zum Gestalt-Ansatz endet mit einer Kulturanalyse von Thomas Bachmann über den Film „Good Will Hunting“ am Ende des Heftes. Darin wird an einem praktischen Fall eine beraterische Intervention untersucht, bei der der Therapeut nach dialogischen Prinzipien des Gestaltansatzes handelt.

Neben diesen Beiträgen zum Themenschwerpunkt finden sich nun einige weitere Texte zu unterschiedlichen Themen: Sandra J. Diller, Jonathan Passmore, Hazel Brown, Siegfried Greif und Eva Jonas untersuchen den Zusammenhang von Coachingausbildung und Coachingerfahrung mit der Qualität des Coachings. Den Autor/ innen ist es gelungen, in einer großen Stichprobe 2267 Coaches und 754 Personalmanager, die Coaching beauftragen, zu befragen. Die Ergebnisse zeigen, dass eine längere Coachingausbildung durchaus einen Unterschied in Hinblick auf die Coachingergebnisse macht. Nicht hingegen von signifikanter Bedeutung ist die Länge 
der Erfahrung als Coach, ein Ergebnis, dass vor allem den Weiterbildungskandidat/ innen Mut machen kann, sich auf den Markt zu wagen.

Geschlechtergerechtigkeit in Organisationen ist in aller Munde. Diversity verspricht bessere Kooperation und Führungskultur. Dennoch sind die Beharrungstendenzen in Unternehmen, die bisherigen Machtverhältnisse zu zementieren, stark. Günther Mohr thematisiert erfolgreiche Organisationsentwicklung als Antwort auf gesellschaftliche Forderungen am Beispiel des Gesetzes zur Geschlechterquote in Aufsichtsräten. Er kann aufzeigen, dass Reglementierung durchaus zu erfreulichen Resultaten in Hinblick auf die Performance der Unternehmen führt.

Stefan Busse wendet sich der Expert/innen-Organisation Hochschule zu. Diese unterscheidet sich in vielerlei Hinsicht von anderen Organisationen. So orientieren sich ihre Mitglieder häufig eher in Richtung ihrer jeweiligen Fachgesellschaften, finden dort Status und Anerkennung. Nachwuchs in Universitäten soll nicht an die Organisation gebunden werden, sondern möglichst anderenorts Karriere machen, ob im Hochschulbereich oder im Praxisfeld. In Universitäten herrscht oft ein recht abständiges Verhältnis zu Führung vor. Dennoch - so zeigt Busse - können am Beispiel der Hochschule als hybrider Organisation an vielen Stellen exemplarisch Komplexität und Paradoxien aufgezeigt werden, deren Bedeutung für die Beratung durchaus als übertragbar auf andere Organisationen gelten kann.

Dilemmata in der Führungspraxis sind allgegenwärtig. Konzentration nach Innen und gleichermaßen nach Außen, Würdigung des Einzelfalls und dennoch Gerechtigkeit walten lassen, Orientierung vorgeben und dennoch die Innovationspotenziale der Mitarbeitenden heben ... die Liste kann beliebig erweitert werden. Marieke Born, Antonia Drews, Ulrike Bossmann, Julika Zwack und Jochen Schweitzer untersuchten Umgangsstrategien von mittleren Führungskräften mit situativen Zwickmühlen im Krankenhaus. Durch ein gezieltes Dilemma-Kompetenz-Training konnte die individuelle und organisationale Ressourcenbilanz verbessert werden. Die befragten Führungskräfte erwarben dadurch einen reflexiven Umgang mit Dilemmata, der sich organisational positiv auf das Arbeitsklima und die Entscheidungsfähigkeit auswirkte.

In unserem Praxisbericht wird ein interkulturelles Training für geflüchtete Fachkräfte in den Gesundheitsberufen geschildert. Sidra Khan-Gökkaya und Mike Mösko veranschaulichen die Barrieren, die der Arbeitsmarkt trotz aller Klagen über den immensen Personalnotstand für Migrant/innen als Herausforderungen zur Überwindung bereithält. Fehlende Arbeitskräfte können mit einem interkulturellen Coaching, das instruierende Know-How-Aspekte ebenso beinhaltet wie Kompetenzaufbau in den Bereichen Kooperation, interdisziplinäre Zusammenarbeit u.v.m., unterstützt werden. Die Evaluation des Trainings zeigt deutliches Empowerment der Teilnehmenden auch im Umgang mit Rassismus und Scham.

In der Rubrik Diskurs diskutiert Christoph Schmidt-Lellek Perspektiven für das Coaching nach der Corona Krise. Er geht substanziell den Fragen nach, wie die Arbeitswelt sich in Zeiten einer „Neuen Normalität“ verändern wird. Herausforderungen, Be- und Entschleunigung, Ambiguitäten, vieles wird neu zu entwickeln sein. Dabei wird Coaching als Antwort auf die Veränderungen in vielen Lebensbereichen gefragt sein, aber auch das Format Coaching selbst wird sich wandeln (müssen). Die Leser und Leserinnen der OSC können sich auf eine Reihe von Beiträgen in den 
nächsten Heften und im Vorab der Online-first-Veröffentlichungen freuen, in denen wir die Folgen der Pandemie für Organisationsberatung, Coaching und Supervision thematisieren.

Funding Open Access funding provided by Projekt DEAL.

Open Access Dieser Artikel wird unter der Creative Commons Namensnennung 4.0 International Lizenz veröffentlicht, welche die Nutzung, Vervielfältigung, Bearbeitung, Verbreitung und Wiedergabe in jeglichem Medium und Format erlaubt, sofern Sie den/die ursprünglichen Autor(en) und die Quelle ordnungsgemäß nennen, einen Link zur Creative Commons Lizenz beifügen und angeben, ob Änderungen vorgenommen wurden.

Die in diesem Artikel enthaltenen Bilder und sonstiges Drittmaterial unterliegen ebenfalls der genannten Creative Commons Lizenz, sofern sich aus der Abbildungslegende nichts anderes ergibt. Sofern das betreffende Material nicht unter der genannten Creative Commons Lizenz steht und die betreffende Handlung nicht nach gesetzlichen Vorschriften erlaubt ist, ist für die oben aufgeführten Weiterverwendungen des Materials die Einwilligung des jeweiligen Rechteinhabers einzuholen.

Weitere Details zur Lizenz entnehmen Sie bitte der Lizenzinformation auf http://creativecommons.org/ licenses/by/4.0/deed.de. 